\title{
Black Hills State University Underground Campus
}

\author{
Brianna J. Mount ${ }^{\mathrm{a}, *}$, Keenan J. Thomas ${ }^{\mathrm{b}, \mathrm{c}}$, Kelsey C. Oliver-Mallory ${ }^{\mathrm{c}, \mathrm{d}}$, Kevin T. Lesko ${ }^{\mathrm{c}}$, \\ Richard W. Schnee ${ }^{\mathrm{e}}$, Reyco Henning ${ }^{\mathrm{f}, g}$, Ryan F. MacLellan ${ }^{\mathrm{h}}$, Marcelo B.B. Guerra ${ }^{\mathrm{a}}$, \\ Matthew Busch $^{g, i}$, Cabot-Ann D. Christofferson ${ }^{j}$, J.F. Wilkerson ${ }^{f, g, k}$, Wenqin $\mathrm{Xu}^{\mathrm{f}, g, \mathrm{~h}}$, \\ Dongming $\mathrm{Mei}^{\mathrm{h}}$
}

a School of Natural Sciences, Black Hills State University, Spearfish, SD 57799, USA

b Department of Nuclear Engineering, University of California-Berkeley, CA 94720, USA

c Physics Division, Lawrence Berkeley National Laboratory, CA 94720, USA

d Department of Physics, University of California- Berkeley, CA 94720, USA

e Department of Physics, South Dakota School of Mines and Technology, Rapid City, SD 57701, USA

${ }^{\mathrm{f}}$ Department of Physics and Astronomy, University of North Carolina, Chapel Hill, NC 27514, USA

g Triangle Universities Nuclear Laboratory, Durham, NC 27708, USA

h Department of Physics, University of South Dakota, Vermillion, SD 57069, USA

${ }^{\mathrm{i}}$ Department of Physics, Duke University, Durham, NC 27708, USA

${ }^{\mathbf{j}}$ Department of Chemistry and Applied Biological Sciences, South Dakota School of Mines and Technology, Rapid City, SD 57701, USA

k Oak Ridge National Laboratory, Oak Ridge, TN 37831, USA

\section{A R T I C L E I N F O}

\section{Keywords:}

Low background counting

Dark matter

Neutrino

Gamma ray spectroscopy

\begin{abstract}
A B S T R A C T
The Black Hills State University Underground Campus (BHUC) houses a low background counting facility on the 4850' level of the Sanford Underground Research Facility. There are currently four ultra-low background, highpurity germanium detectors installed in the BHUC and it is anticipated four more detectors will be installed within a year. In total, the BHUC will be able to accommodate up to twelve detectors with space inside a class 1000 cleanroom, an automated liquid nitrogen fill system, on-site personnel assistance and other required utilities.
\end{abstract}

\section{Introduction}

Rare event searches, including direct detection of dark matter and neutrinoless double beta decay, located in underground laboratories around the world require materials with lower and lower concentrations of radioactive elements (Agnes, 2015, 2016; Akerib, 2014; Dell'Oro et al., 2016; Xiao, 2015). These low activities must be quantified both for the selection of materials in underground experiments and to inform simulations for data analysis. Most of the major underground laboratories, such as Boulby Underground Laboratory, Gran Sasso National Laboratory and SNOLAB house low background counting facilities, which are crucial to the success of their large-scale dark matter and neutrino experiments (Lesko, 2015; Lin, 2015; Ghag, 2015). Such a facility has been developed at the Sanford Underground Research Facility (SURF) in the former Homestake mine in South Dakota, which currently hosts low background experiments such as the maJoRAna demonstrator (mJD) and the Large Underground Xenon (LUX) experiment, with future experiments being developed over the next several years with LUX-ZEPLIN (LZ) and the Deep Underground Neutrino Experiment (DUNE) (Heise, 2015).

The Black Hills State University Underground Campus (BHUC) is a multidisciplinary facility located on the $4850^{\prime}$ level of SURF with $\sim 4200 \mathrm{~m}$ water equivalent (m.w.e.) shielding from cosmic rays. The facility is comprised of two separate cleanroom spaces and an outside staging area for small projects and other research and development activities. $55 \mathrm{~m}^{2}$ of class 1000 cleanroom space in this facility is reserved for low background counting stations and related equipment. By providing a centralized location in one of the deepest underground facilities in the world, with critical support such as an automated liquid nitrogen $\left(\mathrm{LN}_{2}\right)$ system and on-site personnel, the low background facility in the BHUC will support rare event physics experiments as well as other ultra-low background assay needs in the science community. The facility is open to all experimental users, not just those hosted by SURF.

\footnotetext{
* Corresponding author

E-mail address: brianna.mount@bhsu.edu (B.J. Mount).
} 


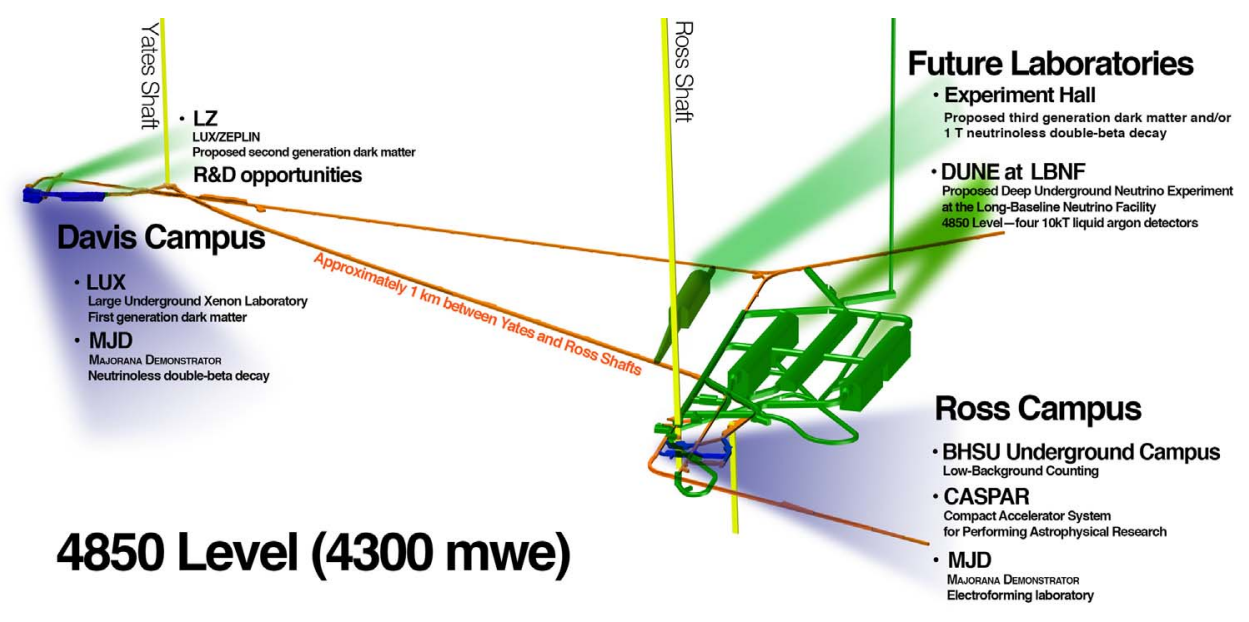

Fig. 1. 4850' level of SURF. Photography courtesy of SURF.

\section{BHUC location and construction}

The BHUC is located on the $4850^{\prime}$ level of SURF, the level at which the major physics experiments are housed (see Fig. 1). The BHUC cavern is within the Ross campus, near the Majorana electroforming lab and the location of the CASPAR experiment. No excavation was necessary as the facility is housed in a renovated cavern.

The walls of the former drill repair shop were stabilized with extensive ground support consisting of rock bolts, meshing and shotcrete. Over the shotcrete two coats of Loxon ${ }^{\circledast}$ Masonry Coating Block Surfacer were applied, followed by one coat of Macropoxy ${ }^{\circledR} 646$ Fast Cure Epoxy. The epoxy was chosen for its ability to act as a radon barrier (Maas, 1997). While the incoming air to the BHUC is not currently radon-reduced, the epoxy layer was applied such that in the future a radon mitigation system might be installed. The cavity sealants will help reduce locally-sourced radon from diffusing into the laboratory airspace. The ambient concentration of $\mathrm{Rn}$ in the BHUC varies between 50 and $300 \mathrm{~Bq} / \mathrm{m}^{3}$.

Once the cavern walls and ceiling were completed, a concrete pad for the cleanroom was poured and a layer of the Macropoxy product was applied to the floor of the cavern. The cleanroom was then constructed. It is comprised of modular panels which are each eight feet high. The $18.5 \mathrm{~m}^{2}$ gowning rooms are soft-walled and the counting room $\left(55 \mathrm{~m}^{2}\right)$ as well as the biology lab $\left(18.5 \mathrm{~m}^{2}\right)$ are hard walled. The construction of the BHUC was finished in September of 2015 and the first counting station was installed that November.

\section{BHUC counting room}

The BHUC counting room is a class 1000 cleanroom. It is anticipated that up to twelve counting stations will be housed in this space. Each station will be supplied with its own dedicated electrical circuit, have access to a 'star' ground and be supplied with network connections. Wireless networks are available throughout the BHUC cavern.

Four $180 \mathrm{~L}$ dewars outside the cleanroom supply each counting station with liquid nitrogen via vacuum-jacketed piping that runs along three walls of the cleanroom. These liquid nitrogen dewars also supply gaseous nitrogen via their boil-off which is in turn also supplied to each counting station as radon purge gas by stainless steel tubing running along the walls.

The liquid nitrogen flow is operated remotely via solenoid valves controlled through LabVIEW software operating a relay board and $\mathrm{LN}_{2}$ level sensor instrumentation. Each $180 \mathrm{~L}$ dewar sits on a scale and the system can monitor these weights (and thus approximate liquid levels). The system also monitors the counting station dewar levels using an American Magnetics, Inc. (AMI) 286 multi-channel liquid level controller, and can pre-cool the room's piping and fill counting station dewars. The solenoid valves may also be controlled by a set of manual switches.

A purged sample storage system is being developed for the BHUC. Once a sample arrives on site, it is placed in a nearly air-tight container and transported underground. Once the purged sample storage system is constructed, the containers will be connected to and stored in a nitrogen environment supplied by the liquid nitrogen boil-off from the external $180 \mathrm{~L}$ dewars. In addition to a shared sample storage system, other support items are shared by users at the BHUC including a wellstocked toolbox, electronics racks with available space and calibration sources.

Black Hills State University (BHSU) faculty and students currently manage and maintain the BHUC, change samples in the detectors and are also available to assist in the installation of detectors underground. BHSU personnel are also available to act as guides for counting room users. Located just $30 \mathrm{~km}$ from SURF, it is relatively easy for BHSU personnel to do same or next-day troubleshooting of counting stations while working with remote user groups.

\section{Current BHUC counting stations}

There are currently four low background counting stations in the BHUC: Morgan, Maeve, Mordred and SOLO (see Fig. 2). Each of these current counting stations are high purity germanium (HPGe) detectors surrounded by $\mathrm{Pb}$ and $\mathrm{Cu}$ shielding and purged with liquid nitrogen

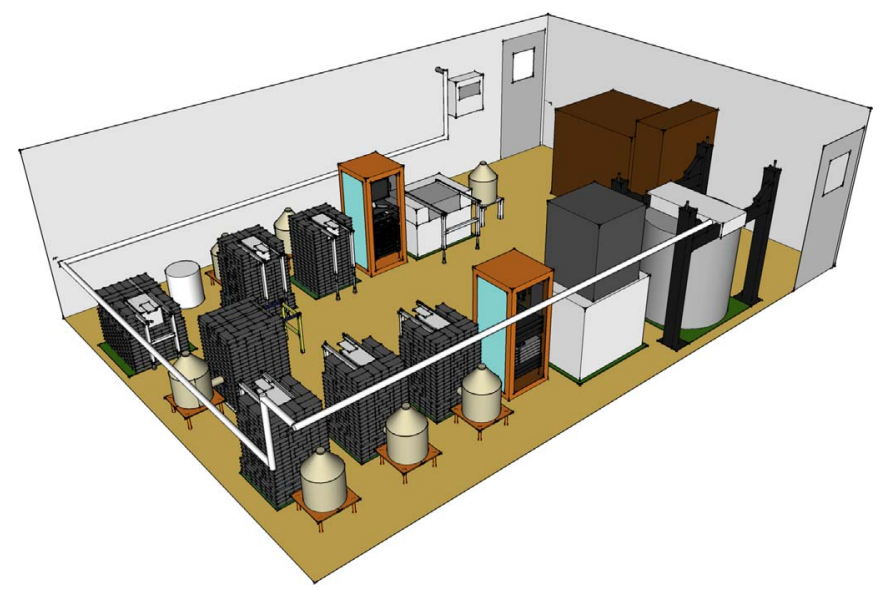

Fig. 2. Current counting stations in the BHUC counting room. From left to right: Mordred, SOLO, Maeve, Morgan HPGe detectors inside their shielding castles. The rack of electronics to the right provides the DAQ and networking for all four detectors. Along the walls of the clean room can be seen the $\mathrm{LN}_{2}$ and $\mathrm{N}_{2}$ lines. The $\mathrm{LN}_{2}$ dewars are located outside the cleanroom to the right of the image. 
boil-off gas.

\subsection{Morgan and Maeve}

The detectors Morgan and Maeve, operated by the Berkeley Low Background Facility (BLBF) of Lawrence Berkeley National Laboratory (LBNL), have a long history with the low background community. The BLBF provides a number of low background gamma-ray spectroscopy and neutron activation analyses to a variety of projects and experiments at a surface low background counting lab at LBNL and a remote site at the BHUC. Prior to the BHUC, detectors were operated underground (600 m.w.e.) at the Hyatt Power Plant in Oroville, California before relocation to SURF (4200 m.w.e.) in 2014. The BLBF has provided support to experiments including SNO, LUX, MAJORANA, CDMS, KamLAND, Daya Bay, Cuore, and many others. Additional information about the BLBF can be found in (Thomas, 2015).

Morgan and Maeve are both ORTEC 80\% p-type HPGe detectors, (2.1 kg and $1.7 \mathrm{~kg}$, respectively) in low background, remote-preamplifier style cryostats. The shields are composed of $20 \mathrm{~cm}$ of Doe Run $\mathrm{Pb}$ and $1.3 \mathrm{~cm}$ of Oxygen-Free High Conductivity (OFHC) $\mathrm{Cu}$, with the exception of Maeve which has an additional $1.3 \mathrm{~cm}$ layer of $\sim 300$ year old $\mathrm{Pb}$.

\section{2. $S O L O$}

SOLO, a $0.6 \mathrm{~kg}$ p-type HPGe detector, was installed in the BHUC in the spring of 2016. Previously, it was located in the Soudan Mine in Minnesota where it was operated by Brown University and Pacific Northwest National Laboratory to provide counting support for a variety of experiments including CDMS, XENON, and LUX (Attisha, 2005). Once relocated to SURF, the shielding was redesigned for the new facility. The inner cavity of the shield is surrounded by $5 \mathrm{~cm}$ of 19th century low activity "German" $\mathrm{Pb}$ and an additional $20 \mathrm{~cm}$ of Doe Run $\mathrm{Pb}$ and purged with nitrogen gas. It is currently hosted by the LZ collaboration and will primarily provide screening for the LZ experiment for the next two years.

\subsection{Mordred}

Mordred, a 60\% ORTEC n-type, $1.4 \mathrm{~kg}$ HPGE detector is collaboratively operated by the BLBF and the CUBED collaboration at the University of South Dakota and was installed in the BHUC in July of 2016. This detector has an inner shield composed of $5 \mathrm{~cm}$ of OFHC Cu and $20 \mathrm{~cm}$ of Doe Run $\mathrm{Pb}$, and the inner cavity is purged with nitrogen. Mordred will primarily provide screening for the LZ experiment for the next several years.

\section{Proposed counting stations}

While the currently installed counting stations in the BHUC are all HPGe detectors, the next generation of detectors will increase the suite of capabilities and sensitivity, as well as broaden participation to other large-scale experiments with low background counting stations in the BHUC.

\subsection{Twins}

A dual detector low background counting station is in development among the BLBF/LBNL, UCB, UCSB, and BHSU groups. This detector station will hold dual ORTEC $(2 \times 2.1 \mathrm{~kg}) 100 \%$ p-type HPGe detectors upon the same sample in a large inner chamber $(\sim 30 \mathrm{~L})$ with the ability to reconfigure the positioning of the two detectors along one axis to accommodate large or very small sample geometries situated between and around the detectors. Sensitivity will be bolstered by having additional crystal mass as well as the ability to perform coincidence/ anticoincidence measurements for select applications. The shielding will be composed of $25 \mathrm{~cm}$ of Doe Run $\mathrm{Pb}$ and an inner $2.5 \mathrm{~cm}$ layer of OFHC $\mathrm{Cu}$. The dual detector system will be installed in the BHUC in the Fall of 2016.

\subsection{MALBEK/MAVRACS}

The Majorana Low Background BEGe Detector at KURF (MALBEK) was transported from Kimballton Underground Research Facility (KURF) in Virginia to SURF in June 2016. It is anticipated that it will be installed in the BHUC by the end of 2016. At KURF, MALBEK was employed as a testing station for low-energy backgrounds as well as a proposed Data Acquisition (DAQ) system for the MAJORANA DEMONSTRATOR. It also performed a dark matter search (Giovanetti, 2015). In the BHUC, MALBEK will continue the noise and DAQ studies and once finished, will be transformed into a low background counting station based on technology developed for the MAJORANA DEMONSTRATOR (Abgrall, 2014). This transformed counting station will contain a $0.5 \mathrm{~kg}$ p-type HPGe detector and will be dubbed the Majorana Very-low background Radioassay Counting Station (MAVRACS) and will be available as a community resource to assay samples.

\subsection{Ge IV}

The Ge IV detector will be operated by the University of South Dakota, in collaboration with the University of Alabama (UA) initially for the Next Enriched Xenon Observatory (nEXO) experiment. The Ge IV detector will build on the expertise of UA which operates three additional above-ground counters: Ge I, II, and III. All Ge IV internal detector components and shielding have been prescreened to ensure an exceptionally low background counting station. The final parameters and performance of the p-type Canberra (Belgium) detector will be evaluated at UA when the detector is delivered in August. The counting station will be ready for installation in the BHUC this fall. Similar to MALBEK and unlike the BLBF detectors, the Ge IV cold finger leaves its dewar vertically; its $\mathrm{Cu}$ and $\mathrm{Pb}$ shielding sit on an aluminum table above the dewar.

\subsection{BetaCage}

A group at the South Dakota School of Mines and Technology (SDSMT) is developing a surface screener for alpha- and beta-particles on material surfaces based on a prototype (Ahmed, 2014) constructed with groups at Caltech and University of Alberta. This detector, called the BetaCage, is a neon-gas drift chamber constructed from ultrapure materials. The placement of samples inside the chamber, directly under a multiwire proportional counter, provides low threshold, high detection efficiency, and track reconstruction that should greatly reduce backgrounds. Work to demonstrate the screener's discrimination of backgrounds using the prototype is in progress. The installation of the BetaCage at the BHUC is dependent on funding, but could be as early as 2018. Once underground the sensitivity of the BetaCage is expected to be 0.1 betas $\mathrm{keV}^{-1} \mathrm{~m}^{-2}$ day $^{-1}$ and 0.1 alphas $\mathrm{m}^{-2}$ day $^{-1}$. It is hoped to be 100x better than existing surface screeners (Bunker, 2013).

\subsection{XIA UltraLo-1800}

Adding alpha counting capabilities to the BHUC, the LZ experiment has plans to relocate an existing XIA UltraLo-1800 to the BHUC to initially support the LZ experiment. The XIA is a commercial, large area, proportional counter with excellent sensitivity to alpha decays. It will provide critical surface contamination assays, such as monitoring the deposition of radon daughters on detector materials at various stages of development and installation. The XIA UltraLo-1800 is an advanced proportional counter capable of measuring large sample sizes and well known to the low background community (McNally, 2014). 


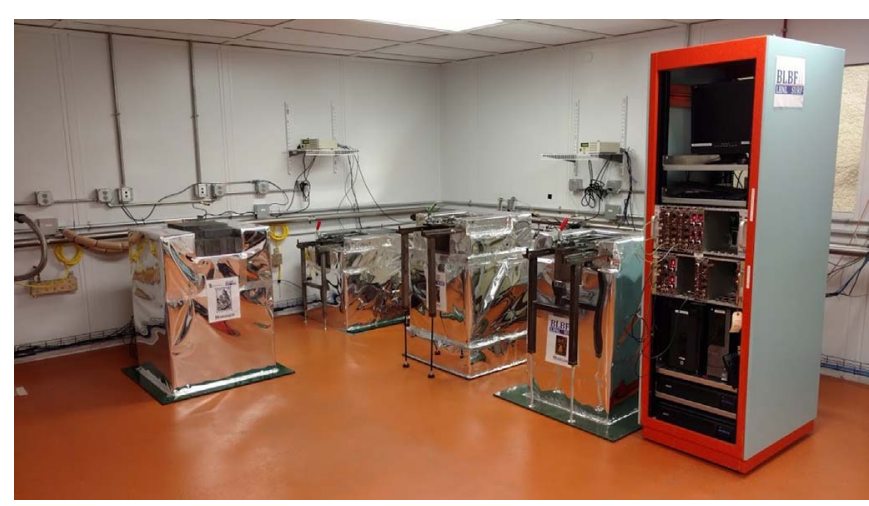

Fig. 3. Current and proposed counting stations in the BHUC counting room.

Table 1

Current, planned and nearby low background capabilities.

\begin{tabular}{lll}
\hline Detector & Detector type & Owner \\
\hline Currently at BHUC & & \\
Morgan & $2.1 \mathrm{~kg}$ p-type, HPGe & BLBF \\
Maeve & $1.7 \mathrm{~kg}$ p-type, HPGe & BLBF \\
Mordred & $1.4 \mathrm{~kg}$ n-type, HPGe & USD/BLBF \\
SOLO & $0.6 \mathrm{~kg}, \mathrm{p}$-type, HPGe & LZ \\
Planned & & \\
Twins & $2 \times 2.1$ kg, p-type, HPGe & BHSU/BLBF \\
MALBEK/MAVRACS & $0.5 \mathrm{~kg}$, p-type, HPGe & MJD \\
Ge IV & p-type, HPGe & nEXO \\
BetaCage & alpha and beta counter & SDSMT \\
Alpha Counter & XIA UltraLo 1800 & LZ \\
Nearby & & \\
BHSU ICP-MS & Agilent 7900 & BHSU \\
\hline
\end{tabular}

\subsection{Future counting stations}

Space for new counting stations is available (see Fig. 3). Interested new detector owners submit a brief selection document to a committee comprised of the BHUC lab director and a representative from each current BHUC detector. The committee determines the eligibility of the new detector for space in the BHUC based on whether an ultra-shielded environment is necessary for the new detector, whether the new detector will cause disturbance to current BHUC users and whether the new detector will increase the capabilities of the BHUC, among other criteria. A summary of current, planned and nearby low background capabilities is summarized in (Table 1).

\section{Counting consortium}

While each of these counting stations provide critical support to high priority experiments (LZ, MJD or nEXO), they are available to all collaborations and academic users. A collaboration of BHUC low background counter owners is being formed to organize and simplify incoming counting requests for the broader scientific community. While each individual counting station would retain autonomy, an agreement will be in place to share counting time on counters with time to spare. While the collaboration is currently concentrated on particle astrophysics samples, it is open to counting samples for any application.

All current BHUC users as well as planned future users have agreed to join this counting consortium. Additionally, a new Inductively Coupled Plasma Mass Spectrometer (ICP-MS) located on the BHSU main campus will participate as well. Assay requests for the counting collaboration will be handled through a website maintained by BHSU.

\section{Summary}

The counting cleanroom in the BHUC is a centralized facility with $\sim 4200$ m.w.e. rock overburden, able to house up to twelve low background counting stations. Currently four counting stations are installed and operating in the BHUC, with four additional HPGe anticipated within the year. The current BHUC users are forming a collaboration to pool resources and provide a single point of access for low background counting at SURF.

\section{Acknowledgments}

This work was supported by an award from the South Dakota Board of Regents as well as South Dakota Science and Technology Authority private funding. This work was also supported in part under the US Department of Energy contract DE-AC02-05CH11231 and DESC0014223 and by National Science Foundation grant PHY-1506033. We gratefully acknowledge the logistical and technical support and the access to laboratory infrastructure provided to us by the Sanford Underground Research Facility and its personnel at Lead, South Dakota. SURF was developed by the South Dakota Science and Technology Authority, with an important philanthropic donation from T. Denny Sanford, and is operated by Lawrence Berkeley National Laboratory for the Department of Energy, Office of High Energy Physics.

\section{References}

Abgrall, N., [The Majorana Collaboration], 2014. Adv. High. Energy Phys. 365432. Agnes, P., et al., [DARKSIDE Collaboration], 2016. Phys. DD. 9393, 081101.

Agnese, R., et al., 2015. Phys. Rev. D. 92.

Ahmed, Z., et al., 2014. JINST 9, 1009P.

Akerib, D.S., et al., [LUX Collaboration], 2014. Phys. Rev. Lett. 112, 091303.

Attisha, M., et al., 2005. AIP Conf. Proc. 785, 75.

Bunker, R., et al., 2013. AIP Conf. Proc. 1549, 132.

Dell'Oro, S., et al., 2016. 2162659, 2016.

Ghag, C., 2015. AIP Conf. Proc. 1672, 020003.

Giovanetti, G., et al., 2015. Phys. Procedia 61, 77.

Heise, J., 2015. J. Phys.: Conf. Ser. 606.

Lesko, K.T., 2015. AIP Conf. Proc. 1672, 020001.

Lin, S.T., Yue, Q., 2015. AIP Conf. Proc. 1672, 020002.

Maas, J.J., Renken, K.J., 1997. Int. Radon Symp.,3,1.1.

McNally, B.D., 2014. NIMA 750, 96.

Thomas, K.J., et al., 2015. AIP ConfProc, 1672, 020004.

Xiao, X., et al., [PandaX Collaboration], 2015. Phys. Rev. D 92, 052004. 